\title{
PAUSAS EM ESQUEMAS ENCADEADOS RAZÃO FIXA, INTERVALO FIXO: SERÁ OTEMPO SENHOR DA RAZÃO?
}

\author{
PAUSING IN CHAINED FIXED RATIO, FIXED INTERVAL SCHEDULES: \\ TIMING OVERRULINGTHE RATIO REQUIREMENT?
}

\author{
Joño Claudio Todorovin e Joyce Paola Teixeira Sobrinho \\ UNIVERSIDADE CATÓLICA DE GOIÁS (UCG) E INSTITUTO DE EDUCAÇÃO SUPERIOR DE BRASÍLIA (IESB)
}

\begin{abstract}
RESUMO
Sete ratos foram submetidos a esquemas encadeados de razão fixa e intervalo fixo em uma câmara para estudos de comportamento operante com duas barras de respostas. Pressões em uma barra produziam, segundo um esquema de razão fixa, um estímulo discriminativo na presença do qual acesso a água era obtido por pressionar outra barra, segundo um esquema de intervalo fixo. Em diferentes condições experimentais, o requisito do esquema de razão fixa foi alterado de 1 para 3,5 e 8 respostas, permanecendo constante um intervalo de $1 \mathrm{~min}$ para o esquema de intervalo fixo. Aumentos no requisito de razão aumentaram a pausa associada ao esquema de razão fixa, como esperado, mas alteraram também as pausas associadas ao esquema de intervalo fixo, que permaneceu inalterado. Os resultados foram interpretados como sendo função da predominância do intervalo de tempo entre apresentações do reforçador primário (água) sobre o tempo fixado pelo estímulo discriminativo associado ao esquema.
\end{abstract}

Palavras-chave: controle pelo tempo, esquemas encadeados, razão fixa, intervalo fixo, água, ratos.

\begin{abstract}
Seven rats were submitted to chained fixed ratio, fixed interval schedules of reinforcement in an operant conditioning chamber with two response bars. A fixed ratio schedule in one bar produced a discriminative stimulus associated with a 1-min fixed interval schedule of primary reinforcement (water) in the second response bar. In different experimental conditions the ratio requirement was changed from 1 , to 3 , to 5 , and to 8 responses while the fixed interval schedule was kept constant. As expected, increases in the fixed ratio requirement resulted in increases in pausing associated with the fixed ratio schedule, but changed also pauses in the fixed interval schedule. Results were interpreted as the dominance of control by time between primary reinforcement presentations over the fixed interval control by the discriminative stimulus.
\end{abstract}

Keywords: timing, chained schedules, fixed ratio, fixed interval, water, rats.

O controle do comportamento pela passagem do tempo é um fenômeno dos mais estudados na psicologia experimental. Staddon e Cerutti (2003) definem esse controle (interval timing) como a covariação entre uma variável dependente, como uma pausa no responder, e uma variável independente, como o intervalo entre reforços em um esquema de intervalo fixo (FI). Lejeune, Richelle e Wearden (2006) lembram a importância do trabalho original de Skinner com esquemas de intervalo fixo para o desenvolvimento dos estudos sobre o controle temporal no comportamento animal (Ferster \& Skinner, 1957; Sherman, 1959) e mostram a importância de três teorias recentes sobre o assunto: Killeen e Fetterman, (1988), Machado (1997) e Dragoi, Staddon, Palmer e Buhusi (2003). Ao longo dos últimos 50 anos, várias teorias e diversas revisões da literatura foram publicadas (e.g., Felton \& Lyon, 1966; Schneider, 1969; Dews, 1970; Dukich \& Lee, 1973; Davison, 1974; Shull, 1979; Lowe \& Wearden, 1981; Lejeune \& Wearden, 1991; Aparício, Lopez, \& Nevin, 1995; Machado, 1997).

\footnotetext{
${ }^{1}$ Endereço para correspondência: SHIN QI 01 Conjunto 9 Casa 11 - 71505-090 Braślia/DF. E-mail: joaoclaudio.todorov@gmail.com
} 
Há inúmeros exemplos de estudos de controle temporal na análise experimental do comportamento e na psicologia experimental como um todo, em que o tempo entre respostas, a duração de cada resposta ou a duração do acesso ao reforçador participam de contingências de reforço, seja como estímulo discriminativo (e.g., Arantes \& Machado, 2008), como definição de resposta (e.g., Bejarano \& Hackenberg, 2007) ou como parâmetro do estímulo reforçador (e.g., Todorov, 1973; Alsop \& Porritt, 2006). Em esquemas FI, a possibilidade de uma resposta ser seguida pelo estímulo reforçador depende da passagem de tempo desde a última apresentação do estímulo reforçador ou desde a apresentação de um estímulo discriminativo (Ferster \& Skinner, 1957; Catania, 1984).

Não há na literatura qualquer comparação entre esses dois possíveis tipos de controle temporal em esquemas de intervalo fixo. Sabe-se mais do comportamento característico de esquemas FI quando o tempo é contado a partir do último reforço. Há outras possibilidades, como esquemas múltiplos FI-extinção, em que o tempo para o próximo reforço é contado a partir do fim do estímulo discriminativo que sinaliza o período de extinção, ou esquemas encadeados, como, por exemplo, de razão fixa (FR) e intervalo fixo (enc FR FI). Reynolds e Catania (1961), estudando o fenômeno do contraste comportamental em esquemas múltiplos, apresentam dados para comparação do desempenho em esquemas FI quando esse componente é antecedido por um reforço recebido em esquema de intervalo variável (VI) e quando é antecedido por um componente que assinala extinção (Reynolds \& Catania, 1961, Figura 1b, p. 388). A pausa característica de esquemas FI é menor quando o tempo do intervalo fixo começa a ser marcado a partir do fim do componente de extinção (apresentação do estímulo discriminativo associado ao FI). Os dados referem-se apenas a dois sujeitos experimentais (pombos) e não permitem concluir se a diminuição nas pausas é devida a controle temporal diferente nas duas condições ou ao contraste comportamental provocado pelas taxas de reforço diferentes nos dois componentes que antecedem o FI.

O presente trabalho foi planejado para verificar a resistência do controle temporal em um esquema FI 60 s componente de esquemas encadeados de razão fixa, intervalo fixo (enc FR FI). Nesse esquema, quando o requisito de respostas do esquema FR é 1, um desempenho característico de FI se estabelece a partir da primeira resposta depois do reforço. Aumentos no requisito da razão devem aumentar a pausa pré-razão, mas não alteram o intervalo de tempo entre a última resposta em FR e o reforço em FI. Se o controle temporal estabelecido pelo início do estímulo discriminativo associado ao FI é idêntico ao controle exercido pela apresentação do reforço positivo, as pausas em FI não devem se alterar quando o requisito de respostas em FR é alterado, mesmo que essa alteração resulte em aumento na pausa pré-razão e em consequente aumento no intervalo entre apresentações do reforço primário.

\section{MÉTOdo}

\section{Sujeitos}

Sete ratos Wistar, machos, adultos, serviram como sujeitos. Eram mantidos em gaiolas individuais, com alimentação à vontade, 
e privados de água 48 horas antes de cada sessão experimental.

\section{Equipamento}

Foram utilizadas quatro câmaras experimentais para ratos da MedAssociates, Modular Test Chamber ENV-008 SN:3318, com duas barras de respostas e acesso à água controlado por mecanismo eletromecânico. $\mathrm{O}$ bebedouro estava localizado no centro da parede direita, entre as duas barras, e ficava mergulhado em uma cuba com água localizada na parte externa da caixa. Quando acionado o bebedouro, uma concha com $0,06 \mathrm{ml}$ de água ficava disponível por 4 s. A lâmpada que produzia iluminação ambiente ficava localizada na parede oposta ao bebedouro. Todos os eventos na câmara experimental foram programados e registrados com o uso de um computador compatível com IBM-PC, interface DIG-700P1 e o aplicativo Windows MedPC (SOF-735).

\section{Procedimento}

Depois da modelagem da resposta de pressão à barra, os animais foram expostos à primeira condição experimental, esquemas encadeados FR 1 FI 60 s. O requisito de razão foi alterado para FR 3, quando todos os sete ratos mostraram estabilidade no desempenho da primeira condição experimental. Em sucessivas condições experimentais, o requisito aumentou para 5 e para 8 , retornando a FR 1 na última condição. As luzes da câmara experimental se apagavam durante o reforço e eram novamente ligadas ao término do requisito dos esquemas de razão. Foram registrados para cada sessão as pausas pré-FR e pré-FI e o número de reforços obtidos.

\section{Resultados}

A Figura 1 mostra pausas no esquema de intervalo fixo de $60 \mathrm{~s}$ como função do requisito de respostas no esquema de razão em esquema encadeado razão fixa, intervalo fixo (enc FR FI). Note-se a escala logarítmica para a variável dependente. Dados individuais de sete ratos: três dos sete animais pararam de responder quando a razão fixa foi aumentada para oito respostas. A Figura 1 mostra claramente que a pausa associada ao esquema FI diminuiu sistematicamente com aumentos no requisito do esquema de razão.

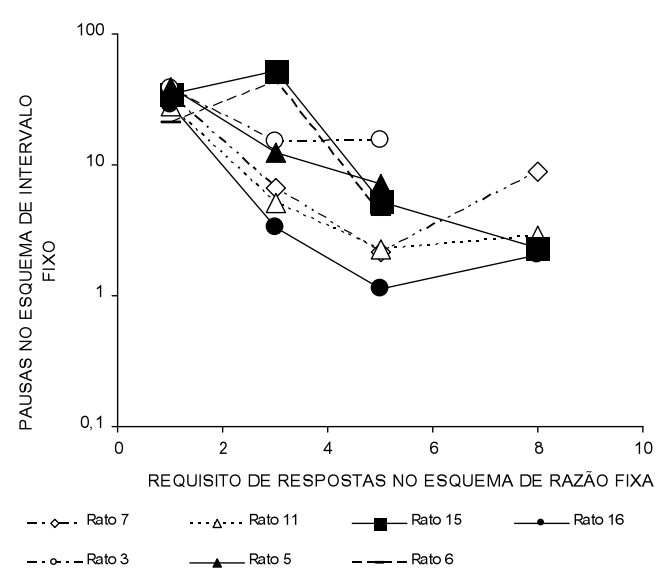

Figura 1. Pausas no esquema de intervalo fixo de $60 \mathrm{~s}$ como função do requisito de respostas no esquema de razão em esquema encadeado razão fixa, intervalo fixo (enc FR FI).

A Figura 2 mostra como aumentaram as pausas no esquema de razão fixa (pós-reforço primário) como função do requisito de respostas no esquema encadeado razão fixa, intervalo fixo (enc FR FI), aumento consistente com o esperado para a relação pausa-requisito de razão (Ferster \& Skinner, 1957). 


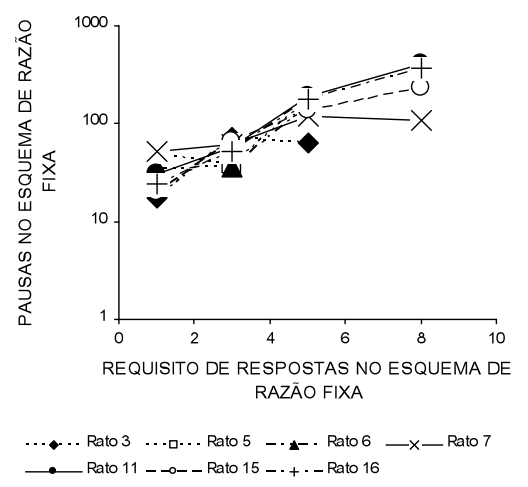

Figura 2. Pausas no esquema de razão fixa (pós-reforço primário) como função do requisito de respostas no esquema encadeado razão fixa, intervalo fixo (enc FR FI). Dados individuais de sete ratos. Note-se a escala logarítmica para a variável dependente. Três dos sete animais pararam de responder quando a razão fixa foi aumentada para oito respostas.

A Figura 3 mostra um efeito inesperado no retorno à linha de base. Depois de longa exposição ao esquema FR 8, para todos os animais a volta a FR 1 significou, a partir da primeira sessão após a última sessão em FR 8, um retorno a pausas iguais às observadas no período de estabilidade da primeira condição experimental.

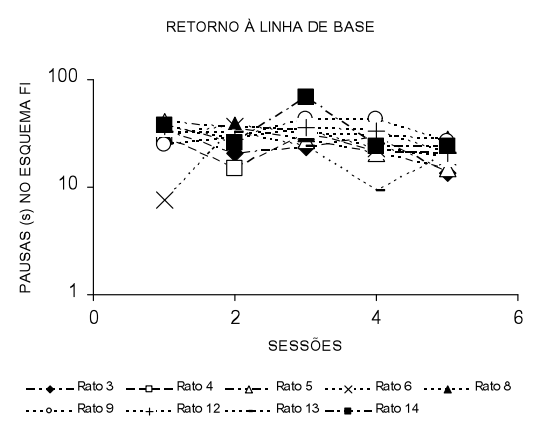

Figura 3. Primeiras cinco sessões no retorno à linha de base (FR 1). As pausas no esquema de intervalo fixo 60 s voltam aos valores iniciais (primeira condição) já na primeira sessão da última condição experimental.

A Figura 4 mostra os dados de estabilidade das pausas associadas ao esquema de intervalo fixo $60 \mathrm{~s}$ nas duas condições experimentais com FR 1 como componente do esquema encadeado (linha de base e retorno à linha de base). Para os sete animais, o retorno significou dados muito semelhantes à condição original, com pouca variabilidade inter e intrassujeitos.

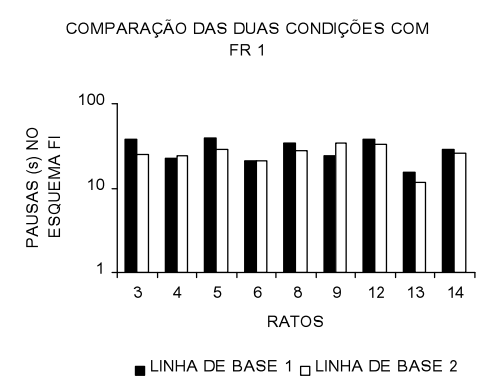

Figura 4. Pausas no esquema Fl na primeira e na última das condições experimentais, ambas com FR 1 como requisito de razão.

A Figura 5 mostra como a pausa no esquema de intervalo fixo mudou com alterações provocadas nos intervalos entre reforços primários causados por aumentos na razão fixa. $\mathrm{O}$ gráfico mostra as pausas como função do número de reforços primários obtidos por hora. Aumentos no requisito de FR produzem aumentos na pausa pré-razão e consequente diminuição da taxa de reforços obtidos por hora.

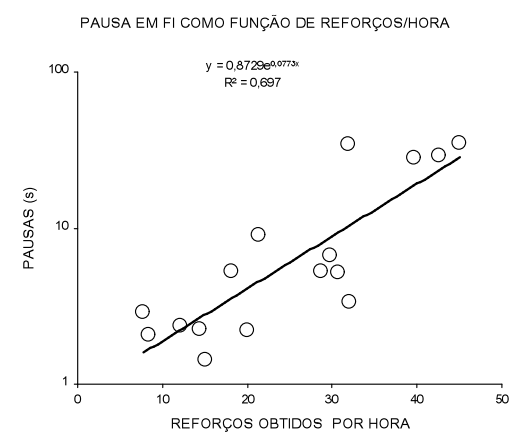

Figura 5. Pausas no esquema de intervalo fixo como função do intervalo real entre reforçadores primários obtidos, expresso como reforços obtidos por hora. Dados agrupados dos ratos 7, 11, 15 e 16. 


\section{DisCUSSÃo}

Os dados do presente experimento mostram claramente que o estímulo discriminativo (luz da câmara experimental) perde o controle sobre a pausa pré-reforço no esquema de intervalo fixo (FI) do esquema encadeado FR FI quando aumentos no requisito do esquema de razão alteram o intervalo entre apresentações do reforçador primário (água). A pausa continua proporcional ao esquema FI, mas o que conta é o intervalo entre reforçadores primários; a luz da caixa perde sua função de estímulo discriminativo.

Os resultados do presente experimento confirmam a observação de Reynolds e Catania (1961) sobre a diminuição das pausas em esquemas múltiplos quando um dos componentes sinaliza extinção se comparado com componentes antecedentes que reforçam a resposta em esquema de intervalo variável, mas levantam dúvidas sobre uma das hipóteses aventadas pelos autores, a que atribui a diminuição das pausas a contraste comportamental. Não seria, também naquele caso, um efeito de mudanças no tempo desde o último reforçador primário, maior quando o componente anterior é de extinção?

Com esses resultados, a definição de esquema de intervalo fixo (e.g., Ferster \& Skinner, 1957; Catania, 1984) merece ser reestudada. Além de outras explorações do esquema encadeado FR FI, outras possibilidades encontradas na literatura são o esquema tandem FR FI estudado por Shull (1970), os esquemas tandem, conjugados e encadeados, explorados por Aparício, Lopez e Nevin (1995) e por Catania, Yohalem e Silverman (1980), e o esquema encadeado FI FR estudado por Hanson e Witoslawski (1959) e Hanson, Campbell e Witoslawski (1962). Em nenhum desses relatos, entretanto, há registro de dados sobre pausas no esquema FI que sejam úteis para a discussão do presente trabalho.

\section{REFERENCIAS}

Alsop, B., \& Porritt, M. (2006). Discriminability and sensitivity to reinforcer magnitude in a detection task. Journal of the Experimental Analysis of Behavior, 85, 41- 56.

Aparício, C. F., Lopez, F., \& Nevin, J. A. (1995). The relation between postreinforcement pause and interreinforcement interval in conjunctive and chain fixed-ratio fixed-time schedules. The Psychological Record, 45, 105-126.

Arantes, J., \& Machado, A. (2008). Context effects in a temporal discrimination task: further tests of the scalar expectancy theory and learning-to-time models. Journal of the Experimental Analysis of Behavior, 90, 33-51.

Bejarano, R., \& Hackenberg, T. D. (2007). IRT stimulus contingencies in chained schedules: Implications for the concept of conditioned reinforcement. Journal of the Experimental Analysis of Behavior, 88, 215-227.

Catania, A. C. (1984). Learning (2nd ed.). Englewood Cliffs, NJ: Prentice-Hall.

Catania, A. C, Yohalem, R, Silverman, P. J. (1980). Contingency and stimulus change in chained schedules of reinforcement. Journal of the Experimental Analysis of Behavior, 5, 167-173.

Davison, M. (1974). A functional analysis of chained fixed-interval schedule performance. Journal of the Experimental Analysis of Behavior, 21, 323-30.

Dews, P. B. (1970). The theory of fixed-interval responding. In W. N. Schoebfeld (Org.). The theory of reinforcement schedules (pp. 43-61). New York, NY:Appleton-Century-Crofts. 
Dragoi, V., Staddon, J. E. R., Palmer, R., \& Buhusi, C. (2003). Interval timing as an emergent learning property. Psychological Review, 110, 126-144.

Dukich, T. D., \& Lee, A. E. (1973). A comparison of measures of responding under fixed-interval schedules. Journal of the Experimental Analysis of Behavior, 20, 281-290.

Felton, M., \& Lyon, D. O. (1966). The postreinforcement pause. Journal of the Experimental Analysis of Behavior, 9, 131-134.

Ferster, C. B., \& Skinner, B. F. (1957). Schedules of reinforcement. New York, NY: Appleton-CenturyCrofts.

Hanson, H. M., Campbell, \& Witoslawski, J. J. (1962). FI length and performance of an FI FR chain schedule of reinforcement. Journal of the Experimental Analysis of Behavior, 5, 331-333.

Hanson, H. M., \& Witoslawski, J. J. (1959). Interactions between the components of a chained schedule. Journal of the Experimental Analysis of Behavior, 2, 171-177.

Killeen, P. R., \& Fetterman, J. G. (1988). A behavioral theory of timing. Psychological Review, 95, 274-295.

Lejeune, H., Richelle, M., \& Wearden, J. H. (2006). About Skinner and time: behavior-analytic contributions to research on animal timing. Journal of the Experimental Analysis of Behavior, $85,125-142$.

Lejeune, H., \& Wearden, J. H. (1991). The comparative psychology of fixed-interval performance: Some quantitative analyses. Learning and Motivation, 22, 84-111.

Lowe, C. F., \& Wearden, J. H. (1981). Weber's law and the fixed-interval post-reinforcement pause. Behavior Analysis Letters, 1, 27-37.
Machado, A. (1997). Learning the temporal dynamics of behavior. Psychological Review, 104, 241-265.

Reynolds, G. S., \& Catania, A. C. (1961). Behavioral contrast with fixed-interval and low-rate reinforcement. Journal of the Experimental Analysis of Behavior, 4, 387-391.

Staddon, J. E. R., \& Cerutti, D. T. (2003). Operant conditioning. Annual Review of Psychology, 54, 115-144.

Schneider, B. A. (1969). A two-state analysis of fixed-interval responding in the pigeon. Journal of the Experimental Analysis of Behavior, 12, 677-687.

Sherman, J. G. (1959). The temporal distribution of responses on fixed-interval schedules (Tese de doutorado). Columbia University, New York, NY, USA.

Shull, R. L. (1970). A response-initiated fixed-interval schedule of reinforcement. Journal of the Experimental Analysis of Behavior, 13, 13-15.

Shull, R. L. (1979). The post-reinforcement pause: Some implications for the correlational law of effect. In M. D. Zeiler \& P. Harzem (Orgs.). Advances in analysis of behavior: Reinforcement and the organization of behavior (Vol. 1, pp. 193-222). New York, NY: Wiley.

Staddon, J. E. R., \& Cerutti, D. T. (2003). Operant conditioning, Annual Review of Psychology, 54, 115-144.

Todorov, J. C. (1973). Interaction of frequency and magnitude of reinforcement on concurrent performances. Journal of the Experimental Analysis of Behavior, 19, 451-458. 